\title{
ACUPUNTURA: BASES CIENTÍFICAS E APLICAÇÕES
}

\author{
ACUPUNCTURE: SCIENTIFIC BASIS AND APPLICATIONS
}

\author{
Márcia Valéria Rizzo Scognamillo-Szabó ${ }^{1}$ Gervásio Henrique Bechara ${ }^{2}$
}

\section{- REVISÃo BIBLIOGRÁFICA -}

\section{RESUMO}

A acupuntura visa à terapia e à cura das enfermidades pela aplicação de estímulos através da pele, com a inserção de agulhas em pontos específicos. Essa técnica esteve isolada do mundo ocidental durante milênios, distanciando sua forma de raciocínio e linguagem da cultura ocidental. Isto restringe sua aceitação no Ocidente, sendo considerada mística e sem base científica. Além disso, a prática da acupuntura no Ocidente se depara com deficiências no ensino e difusão científica. Porém, a eficácia dessa terapia levou a Organização Mundial de Saúde a listar enfermidades que podem ser tratadas pela acupuntura e, recentemente, essa técnica foi reconhecida como especialidade médica veterinária no Brasil. A pesquisa da acupuntura revestese, portanto, de grande interesse, na medida em que poderá traduzir conhecimentos milenares, contribuindo para sua aceitação e incorporação.

Palavras-chave: acupuntura, história da acupuntura, processo inflamatório, inflamação neurogênica.

\section{SUMMARY}

Acupuncture is used for the therapy and illness cure through the application of stimuli in the skin. This is obtained by inserting needles into cutaneous specific points. Such technique was omitted from the occidental world for millennia partially due to its own reasoning and style, which limits the acupuncture to be accepted in the Occident. In addition, the acupuncture practice in the Occident faces a lack of instruction and scientific diffusion. However, the efficacy of such therapy guided the World Health Organisation to list diseases that can be treated through acupuncture. Recently, this therapy was recognised as a veterinary medicine speciality in Brazil. Therefore, research in acupuncture is a very important task and a challenge in the sense it can become clearer millennial knowledge, contributing for its better acceptance and approval.

Key words: veterinary acupuncture, acupuncture history, inflammatory process, neurogenic inflammation.

\section{INTRODUÇÃO}

Derivada dos radicais latinos acus e pungere, que significam agulha e puncionar, respectivamente, a acupuntura visa à terapia e cura das enfermidades pela aplicação de estímulos através da pele, com a inserção de agulhas em pontos específicos (WEN, 1989; JAGGAR, 1992; SCHOEN,1993) chamados acupontos. Trata-se também de uma terapia reflexa, em que o estímulo de uma área age sobre outra(s). Para este fim, utiliza, principalmente, o estímulo nociceptivo (LUNDEBERG, 1993).

Entretanto, além do sentido restrito de agulhamento, a palavra acupuntura pode ter sentido mais amplo, o do estímulo do acuponto segundo as várias técnicas disponíveis (agulhamento, alterações de temperatura, pressão e outras). A acupuntura faz parte de um conjunto de conhecimentos teóricoempíricos, a Medicina Tradicional Chinesa (MTC)

${ }^{1}$ Médico Veterinário, doutorando do curso de pós-graduação em Medicina Veterinária, Faculdade de Ciências Agrárias e Veterinárias (FCAV), Universidade Estadual Paulista (UNESP), 14884-902, Jaboticabal, SP, Brasil. E-mail: szabo@asbyte.com.br. Autora para correspondência.

${ }^{2}$ Médico Veterinário, Professor Titular do Departamento de Patologia Veterinária, FCAV, UNESP

Recebido para publicação em 05.05.00. Aprovado em 27.12.00 
que inclui técnicas de massagem (Tui-Na), exercícios respiratórios (Chi-Gung), orientações nutricionais (Shu-Shieh) e a farmacopéia chinesa (medicamentos de origem animal, vegetal e mineral) (ALTMAN, 1997).

\section{Histórico}

No Oriente, a acupuntura vem sendo usada com finalidades preventiva e terapêutica há vários milênios. De fato, agulhas de pedra e de espinha de peixe foram utilizadas na China durante a Idade da Pedra (cerca de 3000 anos AC). Ney Jing, ou "Clássico do Imperador Amarelo sobre Medicina Interna", texto clássico e fundamental da MTC, descreve aspectos anatômicos, fisiológicos, patológicos, diagnósticos e terapêuticos das moléstias à luz da medicina oriental. Nesse tratado, já se afirmava que o sangue flui continuamente por todo o corpo, sob controle do coração. Cerca de 2000 anos depois, mais precisamente em 1628, William Harvey, proporia sua teoria sobre a circulação sangüínea (ALTMAN, 1992).

A acupuntura veterinária é, provavelmente, tão antiga quanto a história da acupuntura, acompanhando-a pari passu. Estima-se em 3000 anos a idade de um tratado descoberto no Sri Lanka sobre o uso de acupuntura em elefantes indianos. (ALTMAN, 1997).

A introdução da acupuntura no Ocidente está vinculada à fundação da Companhia das Índias Ocidentais, em 1602. Já a acupuntura veterinária se inicia na Escola de Veterinária de Alfort, quando Lepetit e Bernar publicam ilustrações com localização dos canais de acupuntura em cães (SCHIPPERS, 1993).

\section{Os pontos de Acupuntura (acupontos)}

Os acupontos foram empiricamente determinados no transcorrer de milhares de anos de prática médica (RISTOL, 1997). Acuponto é uma região da pele em que é grande a concentração de terminações nervosas sensoriais, Essa região está em relação íntima com nervos, vasos sangüíneos, tendões, periósteos e cápsulas articulares (WU, 1990). Sua estimulação possibilita acesso direto ao SNC (FARBER \& TIMO-IARIA, 1994). Estudos morfofuncionais identificaram plexos nervosos, elementos vasculares e feixes musculares como sendo os mais prováveis sítios receptores dos acupontos. Outros receptores encapsulados, principalmente o órgão de Golgi do tendão e bulbos terminais de Krause também podem ser observados (HWANG, 1992). Diversos trabalhos têm demonstrado grande número de mastócitos nos acupontos. Nesse sentido, Zhai apud HWANG (1992) verificou que ratos adultos possuem contagens de mastócitos significativamente mais altas nos acupontos que em outros locais. Além disso, os acupontos possuem propriedades elétricas diversas das áreas adjacentes: condutância elevada, menor resistência, padrões de campo organizados e diferenças de potencial elétrico (ALTMAN, 1992). Por isso, são denominados pontos de baixa resistência elétrica da pele (PBRP) e podem ser localizados na superfície da pele através de um localizador de pontos. Em ratos, há uma correlação positiva entre o desenvolvimento pós-natal de PBRP e o aumento da contagem de mastócitos no tecido conjuntivo da derme nestes PBRP. KENDALL (1989) verificou que, em acupontos de ratos e humanos, podem ser observadas junções entre mastócitos e fibras nervosas aferentes e eferentes imunerreativas para o neurotransmissor substância P (SP). Segundo HWANG (1992), junções específicas mastócito-célula nervosa foram observadas nos acupontos, bem como relatos de degranulação de mastócitos no acuponto após sua estimulação com agulha. Funcionalmente, os mastócitos estão intimamente relacionados às reações de hipersensibilidade imediata, inflamação neurogênica e enfermidades parasitárias. Devido à gama de estímulos e agentes capazes de ativar o mastócito, tem sido também sugerida sua participação como adjuvante ou amplificador de respostas inflamatórias agudas não relacionadas com hipersensibilidade imediata (Galli apud ABEL, 1995). Sabe-se, por exemplo, que mastócitos produzem interleucina 8 (IL-8), um potente agente quimiotático para neutrófilos (MÖLLER et al., 1993).

A combinação das características descritas tornam o ponto de acupuntura extremamente reativo ao pequeno estímulo causado pela inserção da agulha (KENDALL, 1989).

Segundo GUNN et al. (1976), os acupontos podem ser divididos em tipo I ou pontos motores; tipo II, localizados nas linhas medianas posterior e anterior (ou dorsal e ventral) do organismo e tipo III, que apresentam leitura difusa com neurômetro. Quanto à sua localização, os acupontos dos membros estão situados sobre linhas que seguem o trajeto dos principais nervos e vasos sangüíneos, os do tronco, ao nível da inervação segmentar, local onde nervos e vasos sagüíneos penetram a fascia muscular e os da cabeça e face, próximos aos nervos cranianos e cervicais superiores (KENDALL, 1989). A figura 1 ilustra parte dos acupontos do cão.

\section{Contra-indicações da acupuntura}

É contra-indicado o uso da acupuntura durante a gestação, sobre dermatites ou áreas tumorais e em portadores de marca-passo (BANNERMAN, 


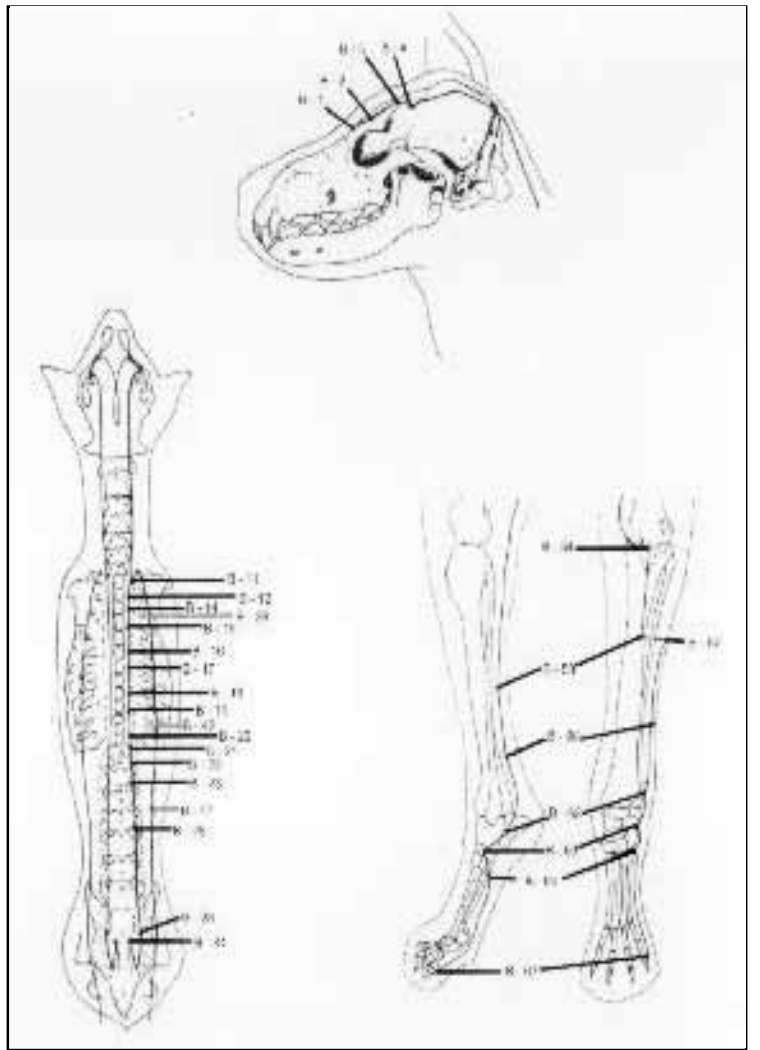

Figura 1 - Canal de energia da bexiga do cão (fonte: BRUNNER, 1981).

1980; STANDARD, 1990). Também é contraindicada a instituição do tratamento com acupuntura antes de elaborado diagnóstico, correndo-se o risco de mascarar ou alterar os sinais clínicos (ALTMAN, 1992).

\section{Incorporação da acupuntura à medicina conven- cional praticada no Ocidente}

Os conhecimentos da acupuntura estiveram isolados do mundo ocidental por cerca de 5000 anos (MANN, 1971; WEN, 1989), distanciando a forma de raciocínio e linguagem. Além do empecilho semântico, a prática dessa técnica se depara com deficiências no ensino e difusão científica (CIGNOLINI, 1990). A ciência rejeita o princípio energético, linguagem metafísica e sistema aparentemente primitivo da MTC, dificultando o engajamento de cientistas na investigação e desenvolvimento da acupuntura (KENDALL, 1989; ANDERSSON, 1993). A necessidade de uma linguagem comum para facilitar o ensino, pesquisa, prática médica e troca de informações ao nível global em acupuntura, levou a Organização Mundial de Saúde (OMS) a criar uma nomenclatura internacional padrão (STANDARD, 1990).
Na China, a acupuntura é utilizada rotineiramente para o tratamento de diversas afecções. A eficácia dessa terapia levou, em 1979, especialistas de 12 países presentes ao Seminário InterRegional da OMS (BANNERMAN, 1979) a publicarem uma lista provisória de enfermidades que podem ser tratadas pela acupuntura e que inclui, dentre outras: sinusite, rinite, amidalite, bronquite e conjuntivite agudas, faringite, gastrite, duodenite ulcerativa e colites agudas e crônicas. Recentemente, a acupuntura foi reconhecida como especialidade médica veterinária em nosso país (BRASIL, 1995). Em Medicina Veterinária, a acupuntura também é indicada para o tratamento de diversas doenças, tais como: gastrites, enterites, colites, bronquite, broncopneumonia, pleurisia, miocardites, arritmia cardíaca, nefrites, alterações na micção, prostatite, cistite, hipotiroidismo, hipertiroidismo, diabetes insipidus, espondilopatia hipertrófica, paralisia facial, epilepsia, sequielas da cinomose, mastite, conjuntivite, otite média, entre outras (ALTMAN, 1997).

\section{Efeito da acupuntura sobre processos fisiopatoló- gicos específicos}

\section{Processo inflamatório}

O processo inflamatório pode ser definido como a reação do tecido vivo vascularizado a uma agressão local (COTRAN et al., 1994) e ocupa um lugar proeminente na medicina pela sua freqüência e importância na origem de grande parte das enfermidades que acometem o ser humano e os animais (CORREA et al., 1970). A princípio, independente de sua causa, o processo inflamatório se caracteriza por uma série de reações de células e tecidos a uma agressão, seguindo um modelo básico e estereotipado que se repete em muitas espécies diferentes. Entretanto a complexidade das alterações bioquímicas, morfológicas e funcionais que têm lugar durante o processo inflamatório, revelam um interessante exemplo de homeostase (PEREZ-TAMAYO, 1986). Por outro lado, o processo inflamatório pode também representar uma agressão aos tecidos e seu controle é desejável em muitas situações, como nas pneumonias, artrites, meningites, dentre outras. Dada a complexidade da reação, o processo inflamatório é ainda tema de numerosas pesquisas no meio científico. Essa complexidade se dá pela interação simultânea no local inflamado de vários componentes orgânicos, locais e sistêmicos, tais como microcirculação sangüínea e linfática, células e fibras do tecido conjuntivo, sistema endócrino e sistema nervoso (ROCHA e SILVA \& GARCIA LEME, 1972).

Neurônios sensitivos periféricos, além de transmitirem sinais aferentes, respondem à estimulação 
elétrica ou estimulação química com um reflexo axônico local desencadeando a liberação de potentes peptídeos vasoativos na área inervada. Essa liberação de peptídeos, que tem sido predominantemente estudada na pele, vias aéreas e articulações de diferentes espécies animais e do ser humano, pode causar vasodilatação local e edema, i.e. inflamação neurogênica (LUNDEBERG, 1993). A inflamação neurogênica é causada pela excitação de fibras aferentes primárias do tipo $\mathrm{C}$ e liberação de mediadores em suas terminações periféricas, levando à contração de musculatura lisa, aumento na permeabilidade vascular, recrutamento de células inflamatórias, degranulação de mastócitos e estimulação de secreção mucosa. As taquicininas SP, neurocinina A (NKA), neurocinina $\mathrm{B}$ (NKB) e, provavelmente, outros peptídeos como o peptídeo relacionado ao gene da calcitonina (CGRP) são mediadores nesse processo. A inflamação neurogênica está presente não só na pele como também em muitos órgãos ocos (vísceras) e pode contribuir para a patologia de várias enfermidades inflamatórias (SARIA \& LUNDBERG, 1995).

A SP, um neuropeptídeo sintetizado na raiz dorsal ganglionar (Harmar et al. apud ABEL, 1995) é transportada axoplasmaticamente até às porções terminais dos nervos sensitivos e sua liberação estimula várias atividades biológicas, entre as quais: contração de musculatura lisa, vasodilatação, secreção glandular e eventos que contribuem com o desenvolvimento da inflamação neurogênica. No ser humano, quando injetada intravenosamente em baixas concentrações, a SP promove aumento do fluxo sangüíneo na pele e na musculatura regional, mimetizando o efeito da estimulação antidrômica (Pernow, apud ABEL, 1995). A administração intradérmica de SP induz ardor, edema e dor local, efeitos que são parcialmente bloqueados pelo composto básico 48/80 ou com anti-histamínicos, indicando que parte dos efeitos da SP estão relacionados à liberação de histamina de mastócitos (Hargermark, apud ABEL, 1995)

$\mathrm{Na}$ literatura científica, correlações entre acuponto e mecanismos de ação da acupuntura têm sido feitos com elementos do processo inflamatório, em especial, aspectos neurogênicos. Como já citado, HWANG (1992) observou junções específicas entre mastócitos e células nervosas nos acupontos.

KENDALL (1989b) e QINGLAN (1991b) citam que, no modelo da injeção de terebentina no pavilhão auricular de coelhos, a ação antiinflamatória da acupuntura resulta em um terço do aumento no volume de exsudato nos animais testes quando comparados aos controles não tratados. Estes autores também verificaram redução no volume de exsudato e na migração de leucócitos.
Para tal, foi necessário que a inervação simpática estivesse intacta.

ZHAO \& ZHU (1992) sugerem que a acupuntura pode ter efeitos diretos na regulação periférica da liberação de mediadores do processo inflamatório e da dor, levando a uma redução da liberação periférica de substância P (SP). MA (1992) observou a diminuição dos níveis de SP em mulheres através do estímulo de agulhamento durante o trabalho de parto.

SIN et al. (1984) obtiveram supressão da pleurisia aguda induzida por carragenina em ratos através da estimulação de um conjunto de acupontos na região torácica dorsal $\left(\mathrm{T}_{1}-\mathrm{T}_{7}\right)$, sugerindo que a escolha dos acupontos sobre dermátomos e miótomos relacionados ao sítio do processo inflamatório pode ser essencial para eficácia dessa terapia. Ainda no mesmo modelo, utilizando-se a eletroacupuntura, diferentes formatos de onda podem produzir efeitos diversos. A supressão do processo inflamatório agudo pode ser obtida com o uso de onda descontínua (15 e 25 ciclos/min), onda denso-dispersa (15 ciclos/min), pulso periódico de onda dispersa $(8 \mathrm{~Hz})$ e densa $(50 \mathrm{~Hz})$. Mas a eletroacupuntura pode induzir efeitos adversos, nesse caso, com o aumento do processo inflamatório agudo através do uso da onda denso-dispersa (25 ciclos/min), e ondas "riple" e "sawtooth" (SIN, 1986).

ZHAO \& ZHU (1990), trabalhando com artrite experimental em ratos, verificaram que a temperatura da pele e o perímetro da articulação no grupo tratado com eletroacupuntura foram marcadamente menores que no grupo controle.

\section{Cicatrização, neovascularização e regeneração}

ABOLAFIA et al. (1985) demonstraram que a eletroacupuntura pode exercer efeito cicatrizante em feridas experimentalmente induzidas na pele de ratos. Animais tratados com eletroacupuntura apresentam uma cicatrização completa, sem contaminação e com tensão superior àquela dos animais controle. Nesse experimento, o grupo controle, que recebeu tratamento com anti-sépticos, apresentou contaminação da ferida experimental.

A atividade da acupuntura sobre neovascularização foi estudada por JANSEN $\boldsymbol{e t}$ al. (1989). Estes autores constataram um aumento no fluxo sangüíneo em flaps músculo-cutâneos de ratos, através da eletroacupuntura. Comparando tratamentos com eletroacupuntura e com os neuropeptídeos SP e CGRP, verificaram que a borda circulatória do flap moveu-se distalmente $66 \%, 31 \%$ e $49 \%$, respectivamente. $\mathrm{O}$ tratamento com o bloqueador simpático reserpina não alterou o fluxo sangüíneo do flap. $\mathrm{O}$ mecanismo pelo qual a eletroacupuntura aumenta o 
fluxo sangüíneo e a sobrevivência do flap ainda é desconhecido. Entretanto, têm sido sugeridas duas possíveis vias: (1) uma inibição de fibras nervosas vasoconstrictoras simpáticas ou (2) a liberação de neurotransmissores nas terminações nervosas periféricas de neurônios sensitivos primários de pequeno diâmetro (fibras $\mathrm{C}$ ou $\mathrm{A} \delta$ ). Estes autores sugerem que a eletroacupuntura possui maior similaridade com os efeitos atingidos quando se injeta nos flaps os neurotransmissores SP e CGRP do que o efeito obtido com o bloqueio dos neurônios vasoconstrictores simpáticos.

MEDEIROS et al. (1995) induziram úlceras gástricas em camundongos através de restrição alimentar ou da administração de indometacina. Todos os animais foram submetidos a 24 horas de jejum e a avaliação de ocorrência de ulcerações gástricas foi feita 6 horas após os tratamentos com (I) indometacina $(10 \mathrm{mg} / \mathrm{kg}$ s.c.), (II) acupuntura, (III) indometacina + acupuntura, (IV) apenas jejum. A estimulação do acuponto Zuzanli com agulhas permanentes fixadas com cola instantânea foi capaz de diminuir as ulcerações provocadas pelo jejum prolongado, mas não aquelas provocadas pelo antiinflamatório não esteroidal. KENDALL (1989b) cita também que a eletroacupuntura no acuponto Zusanli (36E) em ratos pode promover reparo tecidual em úlceras gástricas, reduzindo a área da úlcera.

GAVIOLLE (1999) estudou a ação da acupuntura sobre o fenômeno regenerativo induzido em girinos Rana catesbeiana submetidos à amputação da cauda. Essa autora observou que a morfologia do tecido em regeneração dos girinos que receberam tratamento com acupuntura diferiu significativamente dos animais não tratados.

\section{Resposta imune}

A seguinte afirmação: "A energia vital, dentro, a energia anormal, fora; se a energia anormal é vitoriosa, é porque a energia vital deve estar insuficiente", extraída do Livro de Medicina Interna, escrito há cerca de 2000 anos, indica que os chineses ancestrais já possuíam alguma noção de imunidade (QINGLAN, 1991a).

$\mathrm{O}$ efeito da acupuntura e moxabustão (aquecimento da pele sobre o ponto do acupuntura com auxílio de um bastão de moxabustão Artemisia vulgaris - em brasa) sobre as funções imunológicas está intimamente relacionado ao local da estimulação. Uma diferença significativa é notada quando são estimulados acupontos ou não-acupontos e também entre estímulos dados a diferentes acupontos. É uma característica da acupuntura manter a função imunológica em um estado ótimo, regulando seus mecanismos. Em geral, a acupuntura pode restaurar a homeostase de um organismo, diminuindo hiperfunções e ativando mecanismos em hipofunção. MENG (1992) classifica essa situação como um efeito regulatório de dupla direção, mas essas ações dependem do estado de saúde do organismo em questão.

QINGLAN (1991a) em ampla revisão descreveu que a acupuntura pode exercer efeito sobre a produção de anticorpos. Isso foi demonstrado através do tratamento com acupuntura e moxabustão para diarréia bacteriana em macacos. O grupo tratado desenvolveu anticorpos mais rapidamente, com título em dobro e mais persistente que o grupo controle. Também foi verificado que a eletroacupuntura e moxabustão reduziram o número total de bactérias recuperadas no exsudato peritoneal de coelhos submetidos à peritonite infecciosa experimental, através do aumento de anticorpos fixadores de complementos, opsonizantes e aglutinantes. Em outro experimento observou-se que a moxabustão pode dobrar o título sérico de aglutininas contra bacilo tifóide em coelhos.

$\mathrm{Na}$ mesma revisão sobre acupunturamoxabustão-imunidade, QINGLAN (1991a) destaca que: 1) O número de leucócitos ou a fagocitose podem estar aumentados nas seguintes situações: moxabustão no acuponto Mingmen (04VG) em camundongos e Baihui (20VG) e Shenshu (23B) em coelhos; irradiação de laser de gás carbônico no acuponto Guanyuanshu (04VC) em coelhos e cabras; eletroacupuntura no acuponto Housanli (36E) em cães; 2) o percentual de linfócitos T e a taxa de linfócitos esterase positivos aumentaram em camundongos imunodeficientes submetidos à moxabustão no acuponto Guanyuan e em macacos com diarréia bacteriana submetidos a uma associação de acupuntura com moxabustão; 3) eletroacupuntura no acuponto Neiguan em coelhos eleva as leucinasencefalinas totais no cérebro e sangue, enquanto a capacidade lítica das células NK (natural killer) sobre células alvo K562 também aumenta significativamente; 4) eletroacupuntura em alta potência e por longos períodos pode induzir imunossupressão. Quando ratos são submetidos a essa técnica nos acupontos Housanli (36E) e Sanyinjiao por $30 \mathrm{mi}-$ nutos diariamente, durante 5 dias, é liberado um fator de imunossupressão. Este pode inibir a proliferação de linfócitos $\mathrm{T}$ induzida por concanavalina $\mathrm{A}$ (Con A). O efeito é mais forte no quinto dia, desaparecendo ao décimo dia. Essa imunossupressão não parece estar relacionada ao ACTH e outros hormônios, nem aos receptores opióides.

Segundo o RESEARCH GROUP OF ACUPUNCTURE ANESTHESIA (1979), o efeito da eletroacupuntura no acuponto Hegu ou Zusanli sobre a imunidade celular em setenta pacientes sub- 
metidos a operações cirúrgicas, variou de acordo com o status imunológico de cada paciente. O teste de transformação blástica, o teste sheep eritrocyte (SE) roseta não ativo e o teste SE roseta ativo mostraram que ocorreu estimulação da imunidade celular, principalmente em indivíduos com níveis baixos ou normais desta, enquanto naqueles com altos níveis de imunidade celular, ocorreu um decréscimo. Isso sugere que essa técnica tem um efeito regulatório sobre a imunidade celular.

Segundo JINZHANG et al. (1979), eletroacupuntura $(2 \mathrm{~Hz})$ no acuponto Housanli de coelhos não altera o número de linfócitos, porém aumenta a formação de pregueamento de membrana, indicando presença de movimentação ativa, e também aumenta a taxa de proliferação celular induzida por fito-hemaglutinina.

ZHENYA et al. (1979) observaram a capacidade de formação de roseta ativa e total e de transformação blástica de linfócitos em vinte mulheres com hiperplasia mamária, antes e após o tratamento com acupuntura. Os resultados demonstraram que a acupuntura foi capaz de promover a formação ativa e total de roseta e também a transformação de linfócitos em linfoblastos.

$\mathrm{O}$ estímulo repetido com eletroacupuntura $(2 \mathrm{~Hz})$ no acuponto $36 \mathrm{E}$ de cães sadios levou a um decréscimo nas células alfa-naftil-acetato-esterase positivas (ANAE), no índice de estimulação de linfócitos que sofreram transformação blástica pela incubação com fito-hemaglutinina e nas gamaglobulinas séricas. Isso representa um decréscimo na resposta imunológica basal e, provavelmente, ocorre com envolvimento das células T (KUDO et $\boldsymbol{a l}$., 1987).

KASAHARA et $\boldsymbol{a l}$. (1992) relatam a supressão da reação de hipersensibilidade do tipo tardia (RHTT) ao trinitroclorobenzeno (TNCB) através da acupuntura em camundongos. Estes autores observaram em camundongos BALB/c, C57BL/6 e ddY uma significante supressão da RHTT quando submetidos à acupuntura uma vez ao dia por três dias consecutivos ou após uma única aplicação antes do desafio com TNCB. A aplicação das agulhas na região média do músculo femural (sham-acupuntura) não suprimiu a RHTT ao TCNB.

Em recente estudo sobre o efeito da acupuntura e moxabustão na hipersensibilidade induzida em cobaias e cães por carrapatos da espécie Rhipicephalus sanguineus, SCOGNAMILLOSZABÓ (1999) observou que a moxabustão, aplicada na fase de sensibilização em cobaias, prolongou a reação tardia ao teste cutâneo de hipersensibilidade e diminuiu o número de eosinófilos no local, ambos de forma significativa. Além disso, a acupuntura, realizada durante a manifestação de reação de hipersensibilidade imediata induzida por antígenos do carrapato em cães, foi capaz de diminuir a intensidade da reação de maneira significativa.

\section{Outras aplicações da acupuntura}

Tem sido demonstrado experimentalmente que a colocação de agulhas no filtro nasal de gatos aumenta a resistência ao choque hipovolêmico e que, acupuntura no acuponto 26 do canal do VasoGovernador (26VG) aumenta a oxigenação tecidual em cérebros de ratos. A estimulação deste mesmo acuponto em cães levemente anestesiados com halotano aumenta o débito cardíaco, frequiência cardíaca, pressão arterial média e diminui a resistência periférica total (ALTMAN, 1979).

A acupuntura também foi avaliada em experimentos com estresse agudo por contenção. GUIMARÃES $\boldsymbol{e t}$ al. (1997) avaliaram o efeito da acupuntura nos acupontos 06BP, 36E, 17VC, 06CS, 20VG durante um período de imobilização de 60 minutos em ratos Wistar, utilizando como parâmetros cardiovasculares pressão arterial e freqüência cardíaca e análise de comportamento. Os resultados obtidos sugerem que a acupuntura aplicada durante o estresse agudo por contenção atenua alguns comportamentos envolvidos na reação de luta ou fuga característica do estresse, de maneira independente dos parâmetros cardiovasculares avaliados.

TOUGAS et al. (1992) demonstraram que a acupuntura é capaz de reduzir, durante $30 \mathrm{~min}$, a secreção ácida do estômago em voluntários sadios do sexo masculino.

ASAMOTO \& TAKESHIGE (1992) estudaram o efeito da acupuntura sobre o apetite. Observaram que a implantação de agulhas nos acupontos auriculares correspondentes ao piloro, pulmão, traquéia, estômago, esôfago, sistema endócrino e coração reduziu o ganho de peso em ratos obesos. Segundo estes autores, isso poderia ocorrer pelo efeito da acupuntura exercido sobre o núcleo ventromedial, pois a estimulação de regiões específicas do pavilhão auricular de ratos (aurículo-acupuntura) é capaz de evocar potenciais no núcleo hipotalâmico ventro-medial, o centro da saciedade. FARBER $\boldsymbol{e t}$ al. (1996) avaliaram a utilização da acupuntura auricular como tratamento da obesidade em pacientes humanos. O estímulo dos acupontos auriculares Shen Men, estômago, cárdia, subcórtex (interno) levou à diminuição significativa do peso nas pessoas tratadas, com grandes variações individuais. Estes autores concluem que a acupuntura é moderadamente eficaz como auxiliar no tratamento da obesidade.

Em uma revisão sobre o uso de neuroestimulação para o tratamento de angina pectoris, 
COLQUHOUM (1993) defende o uso regular de estimulação elétrica transcutânea (transcutaneal electrical nerve stimulation - TENS), baseado no fato de essa técnica aliviar os sintomas e melhorar a performance sem induzir a chamada isquemia silenciosa (alívio da dor sem prevenção da isquemia). Estes resultados são observados a curto e longo prazo.

Em pacientes vítimas de acidente vascular cerebral (AVC), a acupuntura é capaz de promover uma melhora funcional mais intensa que os métodos usuais de fisioterapia. JOHANSSON et al. (1993) estudaram 78 pacientes com hemiparesia severa, tanto direita quanto esquerda, e observaram que aqueles que receberam o estímulo sensitivo se recuperaram mais rapidamente e de forma mais intensa que os controles, com uma diferença significativa no equilíbrio, mobilidade, atividades da vida diária, qualidade de vida e dias dispendidos no hospital ou com cuidados de enfermeiras no domicílio.

YAO (1993) estudou o efeito pressor da acupuntura em animais hipotensos. Ratos com hipotensão hemorrágica, ou seja, com pressão arterial média (PAM) correspondente a $60 \%$ do valor controle antes da indução da hemorragia, tiveram a PAM elevada a $80 \%$ da PAM controle, após aplicação de eletroacupuntura. Houve também um aumento significativo da atividade do nervo esplênico, ou seja, inibição da resposta depressora pósestimulatória atribuível à inibição simpática.

A eletroacupuntura é capaz de diminuir os níveis de emocionalidade em camundongos. A aplicação de eletroacupuntura nos acupontos $36 \mathrm{E}$ e 06BP em camundongos observados em campo aberto e em labirinto em cruz elevado aumentou significativamente a porcentagem de locomoção central em relação à locomoção total, indicando um efeito ansiolítico da eletroacupuntura (SILVA $\boldsymbol{e t} \boldsymbol{a l}$., 1996). Comparando as técnicas de estimulação do acuponto Zuzanli (36E) com agulha permanente fixada com cola instantânea ou fazendo a contenção do animal e utilizando agulha simples, os mesmos autores observaram que em camundongos em jejum, normoalimentados ou atropinizados, a técnica da agulha permanente não alterou ou não reduziu de forma significativa o trânsito gastrintestinal. Porém, reduções significativas foram observadas em animais sob contenção e acupunturados (MEDEIROS et al., 1996)

COSTA et al. (1996) avaliaram o efeito da acupuntura sobre a indução de estro em éguas puro sangue inglês virgens que apresentavam retardo no aparecimento de estro na estação de monta. A taxa de prenhez à primeira cobertura foi de $88,88 \%$ para o grupo tratado e de $58,33 \%$ para os animais do grupo controle não tratado. A taxa de prenhez geral, incluindo todas as coberturas foi de $88,85 \%$ para o grupo tratado e de $83,33 \%$ para o grupo controle não tratado. Com os resultados obtidos, estes autores concluíram que a acupuntura é uma alternativa para auxiliar o tratamento de éguas que não apresentam ciclo estral no início da estação de monta.

ALVARENGA et al. (1998, 1998a) avaliaram o efeito luteolítico da aplicação de microdoses (um décimo da dose mínima recomendada) de PGF $2 \alpha$ no acuponto Bai Hui em éguas durante a fase luteínica e verificaram ser a microdose tão eficaz quanto a aplicação da dose convencional por via intra-muscular. Por outro lado, a injeção de água destilada no mesmo ponto não produziu efeito luteolítico (LUNA et al., 1999).

\section{CONCLUSÕES}

Como se observa, apesar da eficácia demonstrada em várias situações, a carência nas bases científicas da acupuntura ou na compreensão de sua linguagem têm restringido seu uso. A pesquisa da acupuntura reveste-se portanto de grande interesse, na medida em que poderá traduzir estes conhecimentos milenares, contribuindo para sua aceitação e incorporação. Ao mesmo tempo, poderá colaborar com avanços na medicina de forma geral, e da neurofisiopatologia em especial. De qualquer forma, o bem-estar humano e animal será beneficiado.

\section{REFERÊNCIA BIBLIOGRÁFICA}

ABEL, M.N.C. Modulação da permeabilidade vascular pela solução hipertônica de cloreto de sódio. São Paulo, 1985. 83p. Tese (Doutorado em Patologia Experimental e Comparada) - Faculdade de Medicina Veterinária e Zootecnia, Universidade de São Paulo, 1995.

ABOLAFIA, A.J.A., SUMANO, H., NAVARRO, R., $\boldsymbol{e} \boldsymbol{t}$ al. Evaluación del efecto cicatrizante de la acupuntura. Veterinaria Mexico, v.16, p.27-31, 1985.

ALTMAN, S. Acupuncture as an emergency treatment. California veterinarian, v.15, n.1, p.6-8, 1979.

Terapia pela acupuntura na clínica de pequenos animais. In ETTINGER, S.J. Tratado de medicina interna veterinária: moléstias do cão e do gato. 3ed. São Paulo: Manole, 1992, V.1, p.454-459.

Acupuncture therapy in small animal practice. The Compendium on Continuing Education for Practicing Veterinarian, v.19, n.11, p.1233-1245, 1997.

ALVARENGA, M.A., FERREIRA, J.C.P., MEIRA, C., et al. Induction of luteolysis in mares utilizing a micro-dose of prostaglandin in the sacral lumbar space (bai hui acupoint). In: ANNUAL INTERNATIONAL CONGRESS ON VETERINARY ACUPUNTURE, 24, 1998a, Taipei. 
Abstracts... Taipei : Scientifc Editions, 1998. p.169-171.

ALVARENGA, M.A., FERREIRA, J.P.C., MEIRA, C., et al. Induction of luteolysis in mares utilizing a micro-dose of prostaglandin $F_{2 \alpha}$ in the sacral lumbar space. Equine Veterinary Science, v.18, n.3, p.167-168, 1998a.

ANDERSSON, S. The functional background in acupuncture effects. S candinavian Journal of Rehabilitation Medicine, suppl 29, p.31-60, 1993.

ASAMOTO, S., TAKESHIGE, C. Activation of the saciety center by auricular acupuncture point stimulation. Brain Research Bulletin, v.99, p.157-164, 1992.

BANNERMAN, R.H. Acupuntura: a opinião da OMS. Revista Saúde do Mundo (OMS), dezembro, p.23-28, 1979.

BANNERMAN, R.H. The World Health Organization viewpoint on acupuncture. American Journal of Acupuncture, v.8, n.3, p.231-235, 1980 .

BRASIL. Resolução 625, 16 mar. 1995. Dispões sobre o Registro de título de especialista no âmbito dos Conselhos Regionais de Medicina Veterinária. Diário Oficial da União, Brasília, v.133, n.77, p.5694, 1995.

CIGNOLINI, ${ }^{\text {a }}$ Problems of teaching and diffusion of chinese acupuncture in Europe. Journal of Traditional Chinese Medicine, v.10, n.1, p.9-12, 1990.

COLQUHOUN, D.M. Eletcrical neuroestimulation for angina pectoris. The Medical Journal of Australia, v.158, n.4 p.440-441, 1993.

CORREA,P., ARIAS-STELLA,J., TAMAYO, R.P. et al. Texto de patología. México : La Prensa Médica Mexicana, 1970. $1282 \mathrm{p}$.

COSTA, M.M., MARTINS, A.V., SANTOS, M.R.C., et al. Utilização da acupuntura veterinária no tratamento de éguas com anestro prolongado. In: CONGRESSO PANAMERICANO DE CIÊNCIAS VETERINÁRIAS, 15 , 1996. Campo Grande, MS. Anais... Campo Grande, 1996. p. 227

COTRAN, R.M., KUMAR, V., ROBBINS, S.L. Robbins: pathologic basis of disease. 5.ed. Philadelphia : Saunders, 1994. 1397p.

FARBER, P.L, TIMO-IARIA, C. Acupuntura e sistema nervoso. Jornal Brasileiro de Medicina, v.67, n.5-6, p.125-131, 1994.

FARBER, P.L., MORAN, C.M., L.I, H.Y., D`ELLIA, F.L.G.M., et al. Acupuntura auricular como auxiliar no tratamento da obesidade: estudo simples-cego, randomizado e placebocontrolado. Revista Médico-Científica de Acupuntura, v.1, n.2, p.5-8, 1996 .

GAVIOLLE, M.C. Modelo experimental para o estudo da interação entre acupuntura e o fenômeno regenerativo induzido em caudas de girinos de Rana catesbiana (SHAW, 1802). São Paulo, 1999. 105p. Tese (Doutorado em Patologia Experimental e Comparada) - Faculdade de Medicina Veterinária e Zootecnia, Universidade de São Paulo, 1999.

GUIMARÃES, C.M., PINGE, C.M.C., YAMAMURA, Y., $\boldsymbol{e t}$ al.
Effects of acupuncture on behavioral, cardiovascular and hormonal responses in restraint-stressed Wistar rats. Brazilian Journal of Medical Biological Research, v.30, n.12, p.24-27, 1997

GUNN, C.C., DITCHBURN, F.G., KING, M.H., et al Acupuncture loci: a proposal for their classification according to their relationship to known neural structures. American Journal of Chinese Medicine, v.4, n.2, p.183-195, 1976.

HWANG, Y.C. Anatomy and classification of acupoints Problems in Veterinary Medicine, v.4, n.1, p.12-15, 1992.

JAGGAR, D. History and basic introduction to veterinary acupuncture. Problems in Veterinary Medicine, v.4, n.1, p.13-15, 1992

JANSEN, G., LUNDEBERG, T., KJARTANSSON, J. $\boldsymbol{e}$ t al Acupuncture and sensory neuropeptides increase cutaneous blood flow in rats. Neuroscience Letters, v.97, p.305-309, 1989.

JINZHANG, Z., ZONGHUI, W., RU,Z. Experimental studies of electroacupuncture on cell-mediated immune response of rabbit. In: NATIONAL SYMPOSIUM OF ACUPUNCTURE, MOXIBUSTION AND ACUPUNCTURE ANAESTHESIA, Beijing, 1979 Abstracts... Beijing : Beijing Agricultural University, 1979. p. 510 .

JOHANSSON, K., LINDGREN, I., WIDNER, H., et al. Can sensory stimulation improve the functional outcome in stroke patients? Neurology, v.43, p.2189-2192, 1993.

KASAHARA, T., WU, Y., SAKURAI, Y., et al. Suppressive effect of acupuncture on delayed type hypersensitivity to trinitrochlorobenzene and involviment of opiate receptors. International Journal of Immunopharmacology, v.14, n.4, p.661-665, 1992.

KENDALL, D.E. A scientific model for acupuncture. Part I American Journal of Acupuncture (California), v.17, n.3, p.251-268, 1989

A scientific model for acupuncture. Part II. American Journal of Acupuncture (California), v.17, n.4, p.342-360, $1989 b$.

KUDO, T., KATO, Y., MASUNO, H., et al. The effect of repeated acupuncture stimulation on canine lynphocyte response. Japanese Journal of Veterinary Science, v.49, n.6, p.1009-1013, 1987

LUNA, S.P.L., JOAQUIM, J.G., MINICHELLI, M., et al. The role of bai hui accupoint for induction of luteolysis in mares. In: ANNUAL INTERNATIONAL CONGRESS ON VETERINARY ACUPUNTURE, 25, 1999. Lexington, Kentucky, EUA. Abstracts... Lexington, 1999. p.P29-P31.

LUNDEBERG, T. Peripheral effects of sensory nerve stimulation (acupuncture) in inflammation and ischemia. Scandinavian Journal Rehabilitation Medicine, suppl. 29, p.61-86, 1993.

MA, H. The effect of acupuncture in the content of substance P in serum of gravida during delivery. Chen Tzu Yen Chiu, v.17, n.1, p.65-66, 1992.

MANN, F. Acupuntura: a antiga arte chinesa de curar. São Paulo : Hemus, 1971. 208p. 
MEDEIROS, M.A., COSTA, E.A., REIS, L.C., et al.. Influência do acuponto Zuzanli na função gastrintestinal em camundongos. In: REUNIÃO ANUAL DA FEDEREÇÃO DE SOCIEDADES DE BIOLOGIA EXPERIMENTAL, 11 1996, Caxambu. Resumos... Caxambu : FESBE, 1995, p.874.

MENG, C. Present status of reserach abroad concerning the effect of acupuncture and moxibustion on immunologic functions. Journal of Traditional Chinese Medicine (Beijing), v.12, n.3, p.211-219, 1992.

MÖLLER, A., LIPPERT, U., LESSMAN, et al. Human mast cells produce IL-8. The Journal of Immunology, v.151, n.6, p.3261-3266, 1993.

PEREZ-TAMAYO, R. Introducíon a la patología. 2. ed. Buenos Aires: Médica Panamericana, 1986. 669p.

QINGLAN, W. Research on animal acupuncture-moxibustion immunity. In: WORLD VETERINARY CONGRESS, 24 1991a, Rio de Janeiro. Anais... Rio de Janeiro, 1991. p.57.

Studies of the regulatory effects of acupuncture on YinYang equilibrium. In: WORLD VETERINARY CONGRESS, 24 1991a Rio de Janeiro. Anais... Rio de Janeiro, 1991. p.59.

RESEARCH GROUP OF ACUPUNCTURE ANESTHESIA Effect of electroacupuncture on cell-mediated immunity in human body. In: NATIONAL SYMPOSIUM OF ACUPUNCTURE, MOXIBUSTION AND ACUPUNCTURE ANAESTHESIA, 1979, Beijing. Resumes... Beijing, 1979. p.510.

RISTOL, E.G.-A. Acupuntura y neurología. Revista de Neurología (Barcelona), v.25, n.142, p.894-898, 1997.

ROCHA e SILVA, M., GARCIA LEME, J. Chemical mediators of the acute inflammatory reaction. Oxford : Pergram, 1972. 425p.

SARIA, A, LUNDBERG, J.M. Neurogenic inflammation. In INFLAMMATORY MEDIATORS - SATELLITE SYMPOSIA OF THE 9TH INTERNATIONAL CONGRESS OF PHARMACOLOGY, 1995, London. Annals... London, 1995. p.73-84

SCHIPPERS, R. The history of veterinary acupuncture and moxibustion outside China. Hannover, 1993. 81p. Thesis (PHD) - Tierärztliche Houchschule (Escola Superior de Veterinária de Hannover), Hannover, 1993.

SCHOEN, A.M. Introduction to veterinary acupuncture scientific basis and clinical aplications. In: ANNUAL CONVENTION OF THE AMERICAN ASSOCIATION OF EQUINE PRACTIONERS, 39, 1993. California. Proceedings... California, 1993. p.39.
SCOGNAMILLO-SZABÓ, M.V.R. Efeito da acupuntura sobre a reação inflamatória imune a carrapatos Rhipicephalus sanguineus (Latreille, 1806) em cobaias (Cavia porcellus) e cães. Jaboticabal, 1999. 115p. Dissertação (Mestrado em Patologia Animal) - Faculdade de Ciências Agrárias e Veterinárias, Universidade Estadual Paulista, 1999.

SILVA, Y.M., TABOSA, A., YAMAMURA, Y., et al.. Efeitos da eletroacupuntura no comportamento de camundongos observados no campo aberto e no labirinto em cruz elevado. In: REUNIÃO ANUAL DA FEDERAÇÃO DE SOCIEDADES DE BIOLOGIA EXPERIMENTAL, 11 1996, Caxambu. Anais... Caxambu, 1996. p.17.

SIN, Y.M. Effect of different waveforms on acute pleurisy during electrical acupuncture. American Journal of Acupuncture, v.14, n.1, p.39-42, 1986.

SIN, Y.M., GWEE, M.S., LOH, M.S. Electric acupuncture on carrageenan-pleurisy: comparative study using various body regions for stimulation. American Journal of Acupuncture, v.12, n.4, p.355-358, 1984.

STANDARD international acupuncture nomenclature: memorandum from a WHO meeting. Bulletin of World Health Organization (Genevre), v.68, n.2, p.165-169, 1990.

TOUGAS, G., YUAN, L.Y., RADAMAKER, J.W., et al. Effect of acupuncture on gastric acid secretion in health male volunteers. Digestive Diseases and Sciences, v.37, n.10, p.1576-1582, 1992 .

YAO, T. Acupuncture and somatic nerve stimulation: mechanism underlying effects on cardiovascular and renal activities. Scandinavian Journal of Rehabilitation Medicine, suppl. 29, p.7-18, 1993.

WU, D.Z. Acupuncture and neurophisiology. Clinical Neurology and Neurosurgery, v.92, n.1, p.13-25, 1990.

WEN, T.S. Acupuntura clássica chinesa. 2.ed. São Paulo: Cultrix, 1989. 225p.

ZHAO, F., ZHU, L. [Therapeutic effects of acupuncture on acute experimental arthritis in rats]. Chen Tzu Yen Chiu, v.15, n.3, p.197-202, 1990.

[Effect of electroacupuncture on neurogenic inflammation] Chen Tzu Yen Chiu, v.17, n.3, p.207-211, 1992.

ZHENYA, M., HONG, C., ZENGXIN, Y. Experimental observations on cellular immunological function under the influence of acupuncture. In: NATIONAL SYMPOSIUM OF ACUPUNCTURE, MOXIBUSTION AND ACUPUNCTURE ANAESTHESIA, 1979, Beijing, Abstracts... Beijing, 1979. p.511. 